\title{
Structure and Dynamics of Extracellular Loops in Human Aquaporin-1 from Solid-State NMR and Molecular Dynamics
}

\author{
Shenlin Wang, ${ }^{1,6, \$}$ Christopher Ing, ${ }^{2,3,6}$ Sanaz Emami,${ }^{1,4,6}$ Yunjiang Jiang, ${ }^{5}$ Hongjun Liang, ${ }^{5}$ \\ Régis Pomès, ${ }^{2,3}$ Leonid S. Brown, ${ }^{1,4}$ Vladimir Ladizhansky ${ }^{1,4}$ \\ ${ }^{1}$ Department of Physics, University of Guelph, Guelph, ON, Canada N1G 2W1 \\ ${ }^{2}$ Molecular Structure and Function, Hospital for Sick Children, Toronto, ON, Canada M5G 1X8 \\ ${ }^{3}$ Department of Biochemistry, University of Toronto, Toronto, ON, Canada M5S 1A8 \\ ${ }^{4}$ Biophysics Interdepartmental Group, University of Guelph, Guelph, ON, Canada N1G 2W1 \\ ${ }^{5}$ Department of Cell Physiology and Molecular Biophysics, School of Medicine, Texas Tech University Health \\ Sciences Center, Lubbock, TX, United States 79430 \\ ${ }^{6}$ Equal contributors \\ ${ }^{\$}$ Current address: Beijing Nuclear Magnetic Resonance Center and College of Chemistry and Molecular \\ Engineering, Peking University, Beijing, China 100871
}

\section{Supporting Information}




\section{Sample preparation}

Uniformly ${ }^{15} \mathrm{~N},{ }^{13} \mathrm{C}$-labeled hAQP1 was produced as described by us previously, see these publications for the detailed protocol ${ }^{1,2}$ Briefly, the expression vector pPICZB-hAQP1-MycHis6 (kindly provided by Frederick Öberg and Kristina Hedfalk, Göteborg University, Sweden) ${ }^{1-3}$ encoding full-length hAQP1 with a C-terminal Myc and 6xHis tags was transformed into the protease-deficient $P$. pastoris strain SMD1168H (Invitrogen) by electroporation. The transformants were grown on yeast peptone dextrose sorbitol plates with zeocin and the resulting colonies were screened for the highest level of hAQP1 production after small scale $(25 \mathrm{ml})$ shake-flasks growth in buffered minimal dextrose (BMD) and buffered minimal methanol (BMM) media, as detected by Western blotting. Cells from the best producing colony were used for large scale growth $(1 \mathrm{~L}$ cultures) in shake flasks with isotopically labeled BMD and BMM (using $0.8 \%\left({ }^{15} \mathrm{NH}_{4}\right)_{2} \mathrm{SO}_{4}$ and $0.5 \%{ }^{13} \mathrm{C}$-labeled glucose for the former and $0.5 \%{ }^{13} \mathrm{C}$-methanol and $0.8 \%\left({ }^{15} \mathrm{NH}_{4}\right)_{2} \mathrm{SO}_{4}$ for the latter). The cells were collected by centrifugation, broken by shaking with glass beads after lyticase treatment, and the membrane fraction was solubilized by $5 \%$ n-octyl- $\beta$-D-glucopyranoside. hAQP1 was purified using $\mathrm{Ni}^{2+}$-NTA resin and reconstituted into lipids (egg PC:brain PS =9:1 w/w, Avanti lipids) at protein/lipid weight ratio of 2 by dialysis.

\section{Experimental methods}

SAXS. For synchrotron SAXS studies, the proteoliposome samples were sealed in quartz capillaries (diameter $\sim 1.5 \mathrm{~mm}$, Hilgenberg GmbH, German) and measured at SSRL (Stanford Synchrotron Radiation Lightsource). Incident synchrotron X-rays from a 8-pole Wiggler was monochromatized $(\lambda=1.37776 \AA)$ and focused using a cylindrical mirror, and the scattered radiation was collected using a Rayonix MX225-HE detector (pixel size $73.2 \mu \mathrm{m}, 3072 \times 3072$ array). A typical radiation time at SSRL is 5 seconds, and each sample was measured 6 times. No radiation damage was observed for all measurements. The 2D SAXS powder patterns were integrated using FIT2D (www.esrf.eu/computing/scientific/FIT2D/), and the sample-to-detector distance was calibrated using silver behenate as a standard. The final SAXS data of individual samples were averaged over 6 different measurements. 


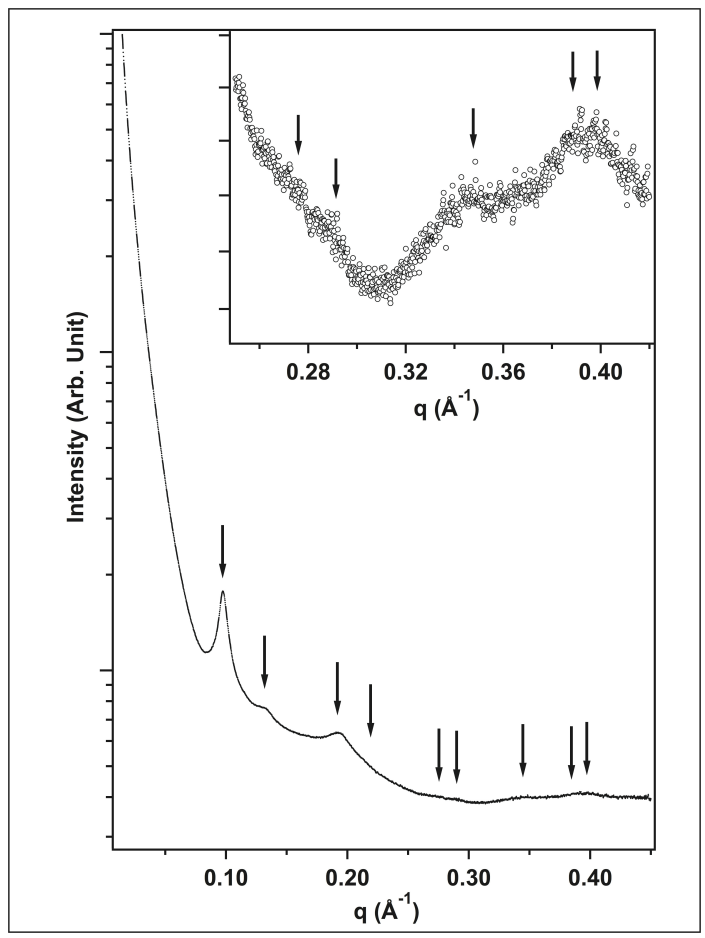

Figure S1. SAXS spectrum of hAQP1 SSNMR sample showing a 2D square lattice (Bragg peaks are shown by arrows). The first 4 peaks show up clearly, and the rest of the peaks at the higher q-range are weak but clearly discernible (inset).

\section{Solid-state NMR}

Proteoliposomes containing approximately $6 \mathrm{mg}$ of UCN hAQP1 were center packed in a 3.2$\mathrm{mm}$ thin wall rotor for NMR experiments. All SSNMR experiments were performed on a Bruker Avance III spectrometer operating at $800.230 \mathrm{MHz}$ equipped with $3.2 \mathrm{~mm}$ EFREE HCN probe (Bruker USA, Billerica, MA).

Typical $90^{\circ}$ pulses were $2.5 \mu$ s for proton, $4 \mu$ s for carbon, and $6 \mu$ s for nitrogen. ${ }^{1} \mathrm{H} /{ }^{15} \mathrm{~N}$ cross-polarization (CP) contact time was $2 \mathrm{~ms}$, with a constant radio-frequency (r.f.) field of 42 $\mathrm{kHz}$ applied on nitrogen, and with a proton field ramped linearly around $\sim 55 \mathrm{kHz}(10 \% \mathrm{ramp}$, optimized experimentally). ${ }^{1} \mathrm{H} /{ }^{13} \mathrm{C} \mathrm{CP}$ contact time was $2 \mathrm{~ms}$, with the constant $\mathrm{r}$.f. carbon field of $64 \mathrm{kHz}$, and with the proton field ramped linearly around $\sim 7 \mathrm{kHz}(10 \% \mathrm{ramp}) .{ }^{15} \mathrm{~N} /{ }^{13} \mathrm{C} \alpha$ and ${ }^{15} \mathrm{~N} /{ }^{13} \mathrm{C}^{\prime}$ band-selective CP steps ${ }^{4}$ were implemented with a contact time of $6 \mathrm{~ms}$. For NCA CP, a constant lock field of $2.5 \times v_{\mathrm{r}}$ ( $v_{\mathrm{r}}$ is the spinning frequency) field strength was applied on ${ }^{15} \mathrm{~N}$, while the carbon field ramped linearly $\left(10 \%\right.$ ramp) around $1.5 \times v_{\mathrm{r}}$. For NCO transfer, a constant lock field of $3.5 \times v_{\mathrm{r}}$ field strength was applied on ${ }^{13} \mathrm{C}$, while ${ }^{15} \mathrm{~N}$ field was ramped linearly $(10 \%$ ramp) around $2.5 \times v_{\mathrm{r}}$. CW decoupling during CP steps was always $90 \mathrm{kHz}$. SPINAL-64 decoupling ${ }^{5}$ of $86 \mathrm{kHz}$ was used in both direct and indirect chemical shift evolutions.

CANCO experiment. 8 scans were recorded with a recycling delay of $1.8 \mathrm{~s}$, resulting in a total acquisition time of $78 \mathrm{~h}$. Data were processed with Lorentzian-to-Gaussian apodization function. 
$16 \mathrm{~Hz}$ of Lorentzian line narrowing and $40 \mathrm{~Hz}$ of Gaussian line broadening were applied in the indirect dimensions, and $40 \mathrm{~Hz}$ of Lorentzian line narrowing and $80 \mathrm{~Hz}$ of Gaussian line broadening were applied in the direct dimension.

NCACX experiments. Two experiments with DARR mixing times of $20 \mathrm{~ms}$ and $50 \mathrm{~ms}$ were recorded. 8 scans per point were recorded, with a recycle delay of $1.7 \mathrm{~s}$. Data were processed with Lorentzian-to-Gaussian apodization functions, with $16 \mathrm{~Hz}$ of Lorentzian line narrowing and $40 \mathrm{~Hz}$ of Gaussian line broadening applied in the nitrogen indirect dimension, and $40 \mathrm{~Hz}$ of Lorentzian line narrowing and $80 \mathrm{~Hz}$ of Gaussian line broadening applied in the carbon dimensions.

3D NCOCX experiments. Two experiments with DARR carbon-carbon mixing times of $50 \mathrm{~ms}$ and $100 \mathrm{~ms}$ were recorded, with 8 scans per point and with a recycle delay of $1.7 \mathrm{~s}$. Data were processed with Lorentzian-to-Gaussian apodization with $16 \mathrm{~Hz}$ of Lorentzian line narrowing and $40 \mathrm{~Hz}$ of Gaussian line broadening in the $\mathrm{t}_{1}$ indirect dimension, and with $40 \mathrm{~Hz}$ of Lorentzian line narrowing and $80 \mathrm{~Hz}$ of Gaussian line broadening in the $t_{2}$ and $t_{3}$ dimensions.

2D NCOCX spectrum was recorded with DARR mixing of $50 \mathrm{~ms}$, and with the acquisition lengths of $14.95 \mathrm{~ms}$ and $20.53 \mathrm{~ms}$ in the indirect $t_{1}$ and direct $t_{2}$ dimensions, respectively. 24 scans per point were recorded with a recycle delay of $1.7 \mathrm{~s}$, resulting in a total acquisition time of $4 \mathrm{~h}$. Data were processed with Lorentzian-to-Gaussian apodization with typical parameters given above.

Carbon chemical shifts were indirectly referenced to 2,2-dimethyl-2-silapentane-5-sulfonic acid (DSS) by adjusting the position of the ${ }^{13} \mathrm{C}$ adamantane downfield peak to $40.48 \mathrm{ppm}{ }^{6}$. Nitrogen chemical shifts were referenced indirectly by using the ratio of gyromagnetic ratios $\gamma_{\mathrm{N}} / \gamma_{\mathrm{C}}=0.402979946$, taken without a temperature factor correction. Experimental data were processed with NMRPipe ${ }^{7}$. Peak picking and noise analysis were performed with the CARA software ${ }^{8}$.

\section{Spectroscopic assignments}

The 3D CANCO experiment establishes interresidue correlations between the backbone nuclei $\mathrm{C}_{\alpha}[\mathrm{i}], \mathrm{N}[\mathrm{i}]$ and $\mathrm{CO}[\mathrm{i}-1]$, and provides nearly full backbone resolution in hAQP1. An example of a 2D NCO plane extracted at the $\mathrm{C}_{\alpha}$ shift of $56.08 \mathrm{ppm}$ is shown in Fig. 2C. The 3D CANCO is complemented by a set of two 3D NCACX intraresidue correlation experiments recorded with DARR (Dipolar Assisted Rotational Resonance) ${ }^{6,9}$ carbon-carbon mixing times of $30 \mathrm{~ms}$ and $50 \mathrm{~ms}$, and two NCOCX interresidue correlation experiments recorded with DARR mixing times of $50 \mathrm{~ms}$ and $100 \mathrm{~ms}$. In both sets, the use of shorter mixing favors one- and twobond correlations (e.g., $\mathrm{N}[\mathrm{i}]-\mathrm{C}_{\alpha}[\mathrm{i}]-\mathrm{CO}[\mathrm{i}] / \mathrm{C}_{\beta}[\mathrm{i}] / \mathrm{C}_{\gamma}[\mathrm{i}]$ or $\mathrm{N}[\mathrm{i}]-\mathrm{CO}[\mathrm{i}-1]-\mathrm{C}_{\alpha}[\mathrm{i}-1] / \mathrm{C}_{\beta}[\mathrm{i}-1]$ in $\mathrm{NCACX}$ and NCOCX, respectively), while longer mixing times result in longer-range intraresidue intercarbon transfers within the carbon chains. It also allows observing interresidue correlations, e.g., $\mathrm{N}[\mathrm{i}]-\mathrm{C}_{\alpha}[\mathrm{i}]-\mathrm{CO}[\mathrm{i}-1]$ and $\mathrm{N}[\mathrm{i}]-\mathrm{CO}[\mathrm{i}-1]-\mathrm{C}_{\alpha}[\mathrm{i}]$ in the NCACX and NCOCX experiments, respectively. An example of a 2D plane of the 3D NCACX experiment is shown in Fig. 2B. Additional information on the side chain shifts was obtained from the $2 \mathrm{D}{ }^{13} \mathrm{C}-{ }^{13} \mathrm{C}$ correlation spectra published previously ${ }^{1}$. 


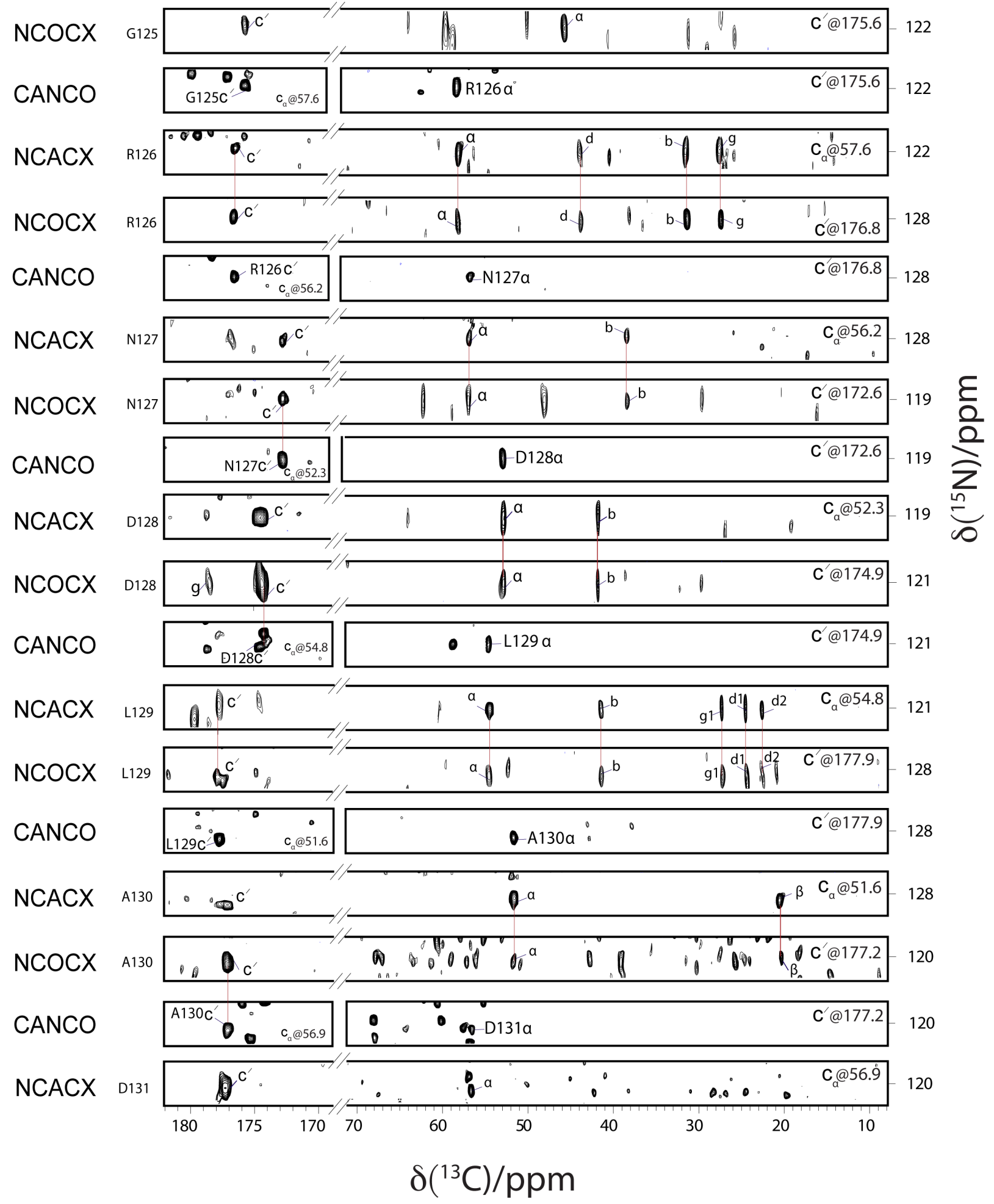

Figure S2. An example of a backbone walk for residues G125-D131 located in loop C. 


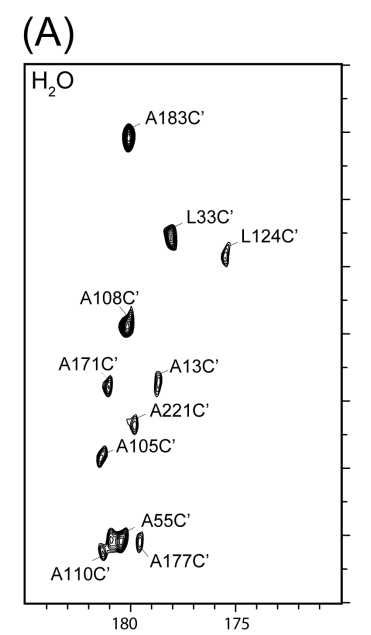

(B)

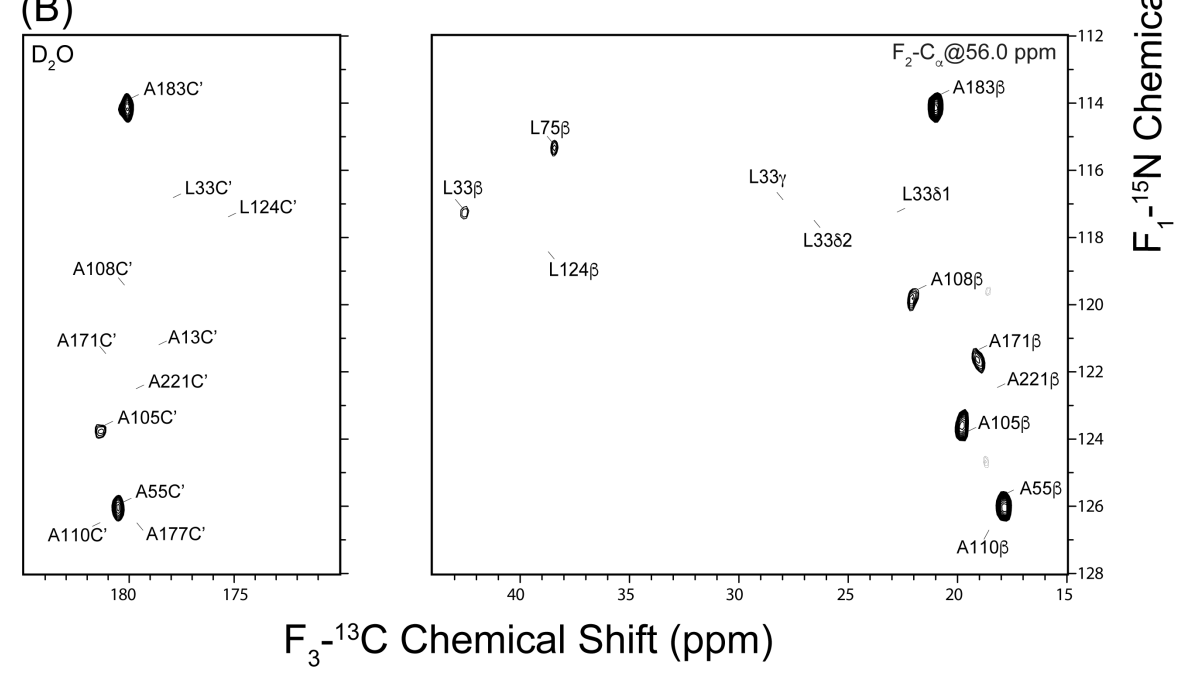

Figure S3. H/D exchange experiments recorded at $800 \mathrm{MHz}$. (A) Two-dimensional N-C plane taken at 56.0 ppm of the three-dimensional NCACX experiment recorded on hAQP1 in the $\mathrm{H}_{2} \mathrm{O}-$ based buffer. (B) The same plane recorded in the $\mathrm{D}_{2} \mathrm{O}$-based buffer. Many peaks corresponding to residues with exchangeable amide protons have attenuated intensities, or disappear completely, e.g., the signals of L33 and A221 clearly detectable in (A) completely disappear after incubation in $\mathrm{D}_{2} \mathrm{O}(\mathbf{B})$, while signals of A171, L75, and A108 are strongly attenuated.

\section{Molecular Dynamics Simulations - Methods}

Homology Modelling. The $3.8 \AA$ electron microscopy (EM) structure of human Aquaporin 1 (PDB: 1FQY) ${ }^{10}$ was used as a template for building homology models with greater agreement to experimental data. De novo modeling of extracellular loops was enforced by removing loop A (residues 37-48) and Loop C (residues 116-136) from the template structure. 
The DOPE potential loop model was used with additional NMR-derived dihedral restraints within MODELLER 9.11 ${ }^{11}$. TALOS+ predicted torsions of "Good" quality were added as additional dihedral energy terms with a force constant derived from a standard deviation of half the value predicted by TALOS + . A cis peptide bond was enforced at the residue 37 to 38 linkage to match experimental predictions in medoids A-E. The trans peptide bond was not modified in simulations of the Aquaporin 1 EM structure models (XTAL).

200 models of the hAQP1 monomer were generated and each of these was used to generate 10 models with loop-only refinements. Of the 1108 successfully generated models, the 56 models with the lowest MODELLER objective function score (the structures with minimum total restraint violations) were then selected for K-Means Coordinates Root Mean-Square clustering implemented in ClusCo ${ }^{12}$. The $\mathrm{C} \alpha$ RMSD was calculated for all pairs of top scoring models. The 56 models were clustered into five groups with populations $36 \%, 20 \%, 18 \%, 16 \%$, $10 \%$ and the structure from the input data that best represented the median of each of the five clusters was extracted and visualized (Figure S4A). When aligned on the transmembrane segments of the EM structure, the set of five medoids had an average C $\alpha$ RMSD of $0.45 \AA, 8.2$ $\AA$, and $5.4 \AA$, for the transmembrane helices, loop $\mathrm{A}$, and loop $\mathrm{C}$ residues, respectively. The initial orientations of side chains in these medoids were inherently biased due to the backbone orientation of the EM structure template, but still show substantial differences for important sidechain-sidechain interactions, such as the R195-E142 sidechain terminal carbon distance (4.4 $\AA, 5.4 \AA, 6.4 \AA, 4.7 \AA, 5.2 \AA, 7.1 \AA$, for the crystal structure and medoid A-E models respectively). In medoid $\mathrm{D}$, the N127 side chain was oriented towards the intracellular side, but in all other models, N127 was outfacing towards the bulk solution. As a crude metric for quantifying loop $\mathrm{A}$ and $\mathrm{C}$ proximity in our initial conditions, we measured the N127-N42 $\mathrm{C} \alpha-\mathrm{C} \alpha$ distance for the crystal structure and medoid structures A through $\mathrm{E}(23.1 \AA, 5.5 \AA, 5.2 \AA, 8.4 \AA$, $15.3 \AA, 5.4 \AA$, respectively).

The medoid structures were compared to all available TALOS+ predictions for verification, resulting in $96 \%, 98 \%, 97 \%, 98 \%, 97 \%$ of all 352 torsional restraints satisfied within 2 standard deviations for each of the five models respectively, with the majority satisfied within 1 standard deviation ( $88 \%$ on average). To quantify the average dihedral angle deviations in the loop A and loop C regions, the RMSD in torsional space was computed with respect to "Good" TALOS+ predicted angles. The medoid structures of each cluster had a dihedral angle RMSD of 15.4, 17.3, 14.8, 14.1, 30.1 degrees. To put these models in context, the dihedral angle RMSD of homologous structures were also calculated: human AQP1 EM structure $1 F Q{ }^{10}$ at 59.8 degrees, an alternative human AQP1 EM structure 1 IH5 ${ }^{13}$ at 82.7 degrees, and the bovine AQP1 structure X-ray structure $1 \mathrm{~J}^{2} \mathrm{~N}^{14}$ at 77.9 degrees.

System Preparation. The medoid structures from each cluster were used to construct five separate hAQP1 tetramers by RMS backbone aligning copies of each monomer onto the original hAQP1 structure using VMD ${ }^{15}$. An average RMSD of less $0.5 \AA$ in the alpha-helical regions of each homology model to the EM structure supports the use of this approach to build a tetramer.

Each of these five models, in addition to the EM structure 1FQY, was independently embedded in a solvated POPC lipid bilayer to obtain a total system size of $13.4 \mathrm{x} 14.1 \mathrm{x} 9.1 \mathrm{~nm}$. The software Lambada ${ }^{16}$ was used to determine the hydrophobic surface of the protein and orient it within a pre-equilibrated lipid patch of 288 lipids. The initial coordinates of the POPC (1-palmitoyl-2-oleoyl-sn-glycero-3-phosphocholine) bilayer were obtained from the laboratory of Dr. Jeff Klauda (72 POPC, $303 \mathrm{~K}$, 
http://terpconnect.umd.edu/ jbklauda/research/download.html). The protein was membrane embedded using the g_membed algorithm ${ }^{1,2,17}$. After membrane embedding each model, the system was ionized using genion with additional chloride atoms to balance the total system charge. Each system included 272 POPC molecules, approximately 15800 water molecules, 25 $\mathrm{Na}^{+}$, and $33 \mathrm{Cl}^{-}$for a total of approximately 160000 atoms.

For all six models, steepest-descent energy minimization was performed to ensure forces were less than $1000 \mathrm{~kJ} \mathrm{~mol}^{-1} \mathrm{~nm}^{-1}$. Two replicas were spawned from each of the energy minimized systems with different random initial velocities. For all replicas of all systems, $1 \mathrm{~ns}$ of NVT simulation (constant particle number, constant temperature, constant volume) followed by 1 ns of NPT simulation (constant particle number, constant pressure, constant temperature) was performed with all protein heavy atoms restrained by a force constant of $1000 \mathrm{~kJ} \mathrm{~mol}^{-1} \mathrm{~nm}^{-1}$.

Simulation Details. All simulations were performed with GROMACS 4.6.5 ${ }^{18}$ with a time step of $2 \mathrm{fs}$. The protein, lipids and ions were modeled with the CHARMM36 all-atom force field ${ }^{19,20}$ and the TIP3P model ${ }^{21}$ was used for water molecules. Lennard-Jones interactions were evaluated using a group-based cutoff for separation distances of $1.2 \mathrm{~nm}$. Coulomb interactions were calculated using the smooth particle-mesh Ewald method ${ }^{22,23}$ with a real-space cutoff of $1.2 \mathrm{~nm}$ and a Fourier grid spacing of $0.16 \mathrm{~nm}$. The non-bonded pair-list was updated every $10 \mathrm{fs}$. Simulation in the NPT ensemble was achieved by semi-isotropic coupling to a ParrinelloRahman barostat ${ }^{24}$ at 1 bar with coupling constants of 2 ps. Temperature coupling was included using the Nosé-Hoover thermostat ${ }^{25,26}$ at $300 \mathrm{~K}$ with a collision frequency of $0.5 \mathrm{ps}^{-1}$ for protein, bilayer and aqueous solution coupling groups for increased accuracy. All covalent bonds were constrained with SETTLE ${ }^{27}$ and P-LINCS ${ }^{28}$ for water and other molecules, respectively.

Production simulations for all unrestrained systems consisted of two repeats to a length of $500 \mathrm{~ns}$ for a total of 6 microseconds. Production simulations for all restrained systems consisted of two repeats simulated in four blocks (15 ns, $50 \mathrm{~ns}, 150 \mathrm{~ns}, 300-350 \mathrm{~ns})$ for a total of 6 microseconds.

Unrestrained Simulation Validation. Average root-mean-square deviation from the EM structure was computed as a function of simulation time to quantify the stability of our protein models (Figure S4B). Large structural deviations were observed in both loop A and loop C for all models, which did not converge on the timescale of our simulations. The structural fluctuations of transmembrane helices converged to an RMSD of $2 \AA$ after 100-150 ns. In subsequent analysis using the unrestrained dataset, $150 \mathrm{~ns}$ was removed in order to reduce the effect of this slow structural equilibration. Data after $150 \mathrm{~ns}$ is referred to as the "unrestrained dataset".

To further assess the quality of models at reproducing TALOS+ restraints, the average angular RMSD to the target TALOS+ predictions was computed as a function of time (Figure S5A-C). The majority of models maintained a lower angular RMSD than that of the EM structure simulations. However, the low angular RMSD values obtained directly after homology modelling (less than 30 degrees for all models) were not maintained over the course of simulation, and converged to stable values around 50-75 degrees after approximately $150 \mathrm{~ns}$.

Restrained Simulation Details. Production simulations were repeated with additional torsional restraints on loop A and loop C. Restraints were derived from all TALOS + torsional predictions of "Good" quality and imposed on our models in a sequence of four simulation blocks (DR1, 
DR2, DR3, and DR4) with increasing harmonic restraints based on TALOS+ standard deviations (all torsional restraints utilized are listed in Table S2). Initial restraints were introduced for all systems starting from our $\mathrm{t}=0 \mathrm{~ns}$ structures (DR1), and subsequent restraint blocks were performed in succession (DR2, DR3, and DR4). DR4 simulations were also referred to as "strong restraints".

Restrained Simulation Validation. Average angular RMSD to TALOS+ predictions was computed to verify a decrease in angular deviation over time for DR1, DR2, DR3, and the first half of DR4 (Figure S5D-F). Deviation gradually decreases until convergence is reached in DR4 with all models having between 15-30 degree average deviation. All subsequent analysis on restrained simulations is from the DR4 block only, after removing the initial 75 ns of simulation, referred to as the "restrained dataset".

The effect of restraints in the restrained dataset was quantified for phi/psi angles of loop $\mathrm{A}$ and loop $\mathrm{C}$ by plotting the average phi or psi angle, taking into consideration angular periodicity, for a given residue against the predicted TALOS+ target for that angle (Figure S6A). The average of residual angles to the TALOS target across all angles, indicated by inset text on each scatter plot, decreases by a factor of 2 to 4 in loop A and loop C upon introducing restraints. This metric may misrepresent the deviation of some residues that sample more than one phi/psi basin over the course of simulation.

The total agreement of TALOS+ restraints was computed by calculating the product of weight factors for each phi or psi angle of loop $\mathrm{C}$ in all simulations. For each monomer of the unrestrained or restrained datasets at all timepoints $(\mathrm{t})$, all phi and psi angles $\left(\mathrm{x}_{\mathrm{i}}\right)$ were assigned an exponential weight factor $\left(\mathrm{W}_{\mathrm{t}}\right)$ between 0 and 1 , based on their deviation to TALOS + target $\left(\mathrm{x}_{\mathrm{i} 0}\right)$ along with the harmonic spring constant restraints imposed in simulations ( $\mathrm{k}_{\mathrm{i}}$, Table S2), for each simulation frame,

$$
W_{t}=\prod_{i} e^{\frac{-k_{i}\left(x_{i}-x_{i 0}\right)^{2}}{2 k_{B} T}}
$$

The distribution of these weight factors for each model were computed using unnormalized histograms plotted on a log-X axis (Figure S6B). Unrestrained simulations have low agreement to TALOS + targets on average, and failed to sample the predicted phi/psi angles in almost all simulations ( $\mathrm{W}_{\mathrm{t}}$ is close to 0$)$. Loop $\mathrm{C}$ conformations from restrained simulations were simultaneously able to satisfy the majority of restraints on average ( $\mathrm{W}_{\mathrm{t}}$ is close to 1$) .66 \%, 77 \%$, $48 \%, 89 \%, 69 \%$, and $17 \%$ of frames from restrained simulations had $\mathrm{W}_{\mathrm{t}}$ values larger than $10^{-5}$ (the product of 40 exponential weight values of 0.75 ) for the EM structure and medoid A through medoid E, respectively.

Water Permeability Calculations. All data beyond the initial equilibration (for both unrestrained and restrained datasets) were analyzed using the collective diffusion model. ${ }^{29} \mathrm{~A}$ cylinder of length $10 \AA$ and radius $5 \AA$ was centered in the vicinity of the NPA motif (defined by the center of mass of residues 56, 60, 76, 146, 150, 173, 177, and $192 \mathrm{C} \alpha$ atoms) and was used to track fractional water displacements along the cylinder between time steps spaced 20 ps apart. The quantity $n(t)$, representing the net amount of water permeation as function of time, was segmented into thousands of non-overlapping 300 ps blocks. The mean square displacement $\left\langle n^{2}(t)\right\rangle$ was computed by taking the mean over all 300 ps blocks, separately for each subunit and each repeat. A linear regression was performed on each of the mean square displacements 
datasets in order to estimate a set of water permeability values, $\mathrm{p}_{\mathrm{f}}$, for a given model. For our estimate of permeability, we report the mean of all $p_{f}$ values for a given model $(2$ repeats $\times 4$ subunits $=8$ data points), and estimate the error in $\mathrm{p}_{\mathrm{f}}$ by the standard error of the mean.

Analysis Details. All molecular dynamics trajectories were processed by aligning C $\alpha$ atoms of all transmembrane segments to the EM structure. Error bars were computed as the standard error of mean across all monomers and replicas within a given system. Molecular dynamics analysis was conducted using MDTraj and MDAnalysis software packages ${ }^{30,31}$. For hydrogen bonding calculations, all interactions between polar atoms were considered hydrogen bonds if the donoracceptor distance was less than $3.5 \AA$ and the donor-hydrogen-acceptor angle was less than 60 degrees. Secondary structure analysis was performed using the DSSP algorithm ${ }^{32}$ on frames spaced at $1 \mathrm{~ns}$. All molecular renderings from molecular dynamics analysis was created using $\mathrm{VMD}^{15}$.

Clustering and Solvent Exposure of Loop C Conformations. For the restrained datasets, oneto-all RMSD clustering was performed on $\mathrm{C} \alpha$ atoms of loop $\mathrm{C}$ for individual monomers of all simulation frames spaced 500 ps apart. All structures were aligned on the $\mathrm{C} \alpha$ atoms of the alphahelical segments (see Analysis Details) to the EM structure before RMSD computation was performed. For each of the 40 monomers in the restrained data sets (five homology models, four monomers per model, and two repeats) we extracted a list of RMSD values for each simulation frame to the greater collection of all frames of all homology models. This matrix of features was used for clustering with the K-Medoids algorithm available in MSMBuilder ${ }^{33}$, with 8 clusters and 3 algorithm passes. These cluster centers and several cluster members are visualized (Figure S8). Pairwise RMSD calculations were performed between the medoids of the 8 restrained simulation clusters, and the EM structure (Figure 9A). Note that the medoid structures presented in this clustering analysis are not the same as the original medoid homology models selected during molecular modelling.

Within each cluster of restrained monomers, we extracted hydrogen bonds between both backbone nitrogen and sidechain nitrogen atoms that were studied in H/D exchange experiments (Figure 9B-C, S9). For each nitrogen atom, we computed the percentage of simulation frames where one hydrogen bond was formed to either protein or backbone. The populations of hydrogen bonds involving nitrogen atoms of amine groups (Asn $\mathrm{N}_{\delta}, \operatorname{Arg} \mathrm{N}_{\eta 1}, \operatorname{Arg} \mathrm{N}_{\eta 2}, \operatorname{Gln}_{\varepsilon}$ ) were scaled by half. Depending on the local environment of the nitrogen, a lack of hydrogen bonds may indicate failure to meet the hydrogen bonding cutoffs, non-polar interactions, or burial from any available hydrogen bonding group. In contrast, there are also transient cases where both water and protein hydrogen bonds are formed, and thus in principle, the percent of simulation frames with hydrogen bonds for a given nitrogen may total greater than $100 \%$. To evaluate qualitative agreement with backbone solvent accessibility, we computed the population of frames where two or more backbone nitrogen atoms of residues 130, 133, or 134 were hydrogen bonded to protein simultaneously. The percentage of all cluster frames that were considered buried by using this metric were 70\%,79\%, 72\%, 47\%, 33\%, 0\%, 78\%, and 93\%. Within these clusters, we computed the qualitative agreement with side chain nitrogen atom solvent accessibility of residues H74, N122, R126 ( $\left.\mathrm{N}_{\eta 1}, \mathrm{~N}_{\eta 2}, \mathrm{~N}_{\varepsilon}\right), \mathrm{N} 127, \mathrm{H} 180$, and R195 $\left(\mathrm{N}_{\varepsilon}\right.$ only). For the aforementioned side chain nitrogen atoms H74, N122, R126, H180, and R195 are expected to be exchangeable, thus we extract the number of frames were all of these sidechains have no protein hydrogen bonds formed. In the same step, we also filter the data to extract all 
frames where $\mathrm{N} 127$ has a protein hydrogen bond. The assumption of this analysis is that a protein hydrogen bond is sufficient as a metric to describe shielding of a nitrogen to the solvent, even though this may not be universally true. The subset of cluster frames that matched the backbone accessibility were then processed to compute the sub-population where all eight side chain hydrogen bond criteria was simultaneously matched, resulting in $2 \%, 5 \%, 1 \%, 2 \%, 1 \%$, $11 \%, 3 \%$, and $4 \%$ of the backbone matching frames. While all clusters are shown here, only clusters $1,2,3,4,7$, and 8 were used in the total averages mentioned in the main text.

R195 Conformational Isomerization. All simulations of hAQP1 began with R195 in close proximity to E142 in a state we refer to as the side-facing conformation, as it is found in the 1FQY EM structure (Figure 11A) ${ }^{10}$. Throughout unrestrained and restrained simulations, the side chain of R195 adopted an up-facing state, often hydrogen bonded to residues of loop C. To classify R195 as either side-facing or up-facing, we developed a metric using its $\chi_{1}$ and $\chi_{2}$ angles, and supported this with the signed $\mathrm{C} \alpha$ to $\mathrm{Cz}$ distance vector. Basins within $\chi_{1}$ and $\chi_{2}$ maps in both unrestrained and restrained datasets had comparable populations, indicating that R195 conformations were not significantly altered by loop restraints (Figure S10A-B). The distribution of $\mathrm{C} \alpha$ to $\mathrm{Cz}$ distances in restrained simulations indicate that $60 \%$ and $40 \%$ of monomer conformations were up-facing and side-facing, respectively, on the basis of a $+2.5 \AA$ division between this bimodal distribution. To better delineate distinct conformations of R195, we isolated conformations based on specific $\chi_{1}$ and $\chi_{2}$ basins that were in agreement with average $\mathrm{C} \alpha$ to $\mathrm{Cz}$ distances. The projection of average $\mathrm{C} \alpha$ to $\mathrm{Cz}$ distance vector onto the $\mathrm{R} 195 \chi_{1}$ and $\chi_{2}$ map suggests that $\left(\mathrm{g}^{+}, \mathrm{t}\right)$ and $\left(\mathrm{t}, \mathrm{g}^{+}\right)$conformations represent side-facing and up-facing states, respectively (Figure S10D-E). All interactions between the polar atoms of R195 and neighbouring residues were extracted using hydrogen bonding analysis within each of the two $\chi_{1}$ and $\chi_{2}$ basins studied. Hydrogen bonding percentage was calculated by extracting the number of frames where one or more oxygen atoms of a residue acted as a proton acceptor to one or more nitrogen atoms of R195, normalized by the total number of frames (Figure S10F). Note that hydrogen bonds made to both the backbone and the side-chain nitrogen atoms of R195 were represented in this analysis (made evident by the large percentage of frames where N192 is a binding partner to the backbone amide of R195). Mutually exclusive hydrogen bonds were made in the side-facing and up-facing conformations. No hydrogen bonds were observed between R195 and H180 in our simulations ${ }^{34}$. Using the same protocol described to generate Figure S9 we extracted the percentage of frames where residues of loop $\mathrm{C}$ made hydrogen bonds with protein or water (Figure S10G-H). We observed no significant change in loop C hydration depending on the conformation of R195, and thus conclude that an up-facing state is not strictly necessary for loop $\mathrm{C}$ to adopt a conformation that satisfies H/D exchange predictions. Similarly, there is asymmetry in the solvent accessibility of R195 identified by this analysis, but this is largely independent of loop C conformations.

Sources of Error in Molecular Dynamics Simulations. Molecular dynamics simulations of rhodopsin have suggested that the mobility of its loops are considerably higher than those in its transmembrane regions, requiring multiple long-timescale simulation repeats to reach convergence ${ }^{35}$. Even though our NMR experiments suggest that part of loop A and the whole loop $\mathrm{C}$ in human AQP1 adopt a semi-structured conformation of potentially limited mobility, we sought to employ a similar computational approach to improve sampling and convergence of loop conformations. Despite this, some aspects of our simulations were only in partial agreement 
with experimental data. For example, validating structural predictions from side chain nitrogen solvent accessibility profiles was very challenging using our computational protocol, resulting in a large portion of our data being unusable for comparison with NMR results. In part, this is due to the fact that we did not simulate the dynamics of excess protons, and as such it was not possible to reproduce the prediction of slow exchangeability of the two asparagine residues of the NPA motif, nor arrive at a formally correct measure of solvent accessibility of nitrogen atoms. Our restrained simulations are likely to be biased by the utilization of the 1FQY EM structure as a template for homology model construction. Through the use of this structure, all of our models were initialized with R195 in a side-facing orientation towards E142 (Figure 11, S4A). As such, it is likely that even with multiple microseconds of aggregate MD sampling, we have not achieved proper convergence of the conformational ensemble of R195. This sampling bottleneck may have been further exasperated by the application of restraints that inherently slows the equilibration of our loops. This may have been addressed by performing massive simulation repeats or by cycling restraints on and off throughout simulation. Furthermore, a recent study found that the CHARMM36 protein force field and the CHARMM TIP3P water model, both used in this study, supressed the folding of dynamic model peptides compared to experimental data ${ }^{36}$. As such, it is possible that our choice of force field may promote a higher population of "unfolded" loop conformations, especially given that loops of the de novo models are not expected to be near the native conformation. Lastly, given that some loop $\mathrm{C}$ side chains were found to interact with POPC head groups (e.g. R126), it may be possible that a difference in bilayer composition between experiment (9:1 PC:PS) and simulation (1:0 PC:PS) may result in a source of error when comparing the two studies.

Table S1. Comparison of secondary structure elements in aquaporin 1 derived from NMR, X-ray and EM studies.

\begin{tabular}{clccccc}
\hline & NMR data & $\begin{array}{c}\text { EM } \\
(1 \mathrm{FQY})^{10}\end{array}$ & $\begin{array}{c}\text { EM } \\
(1 \mathrm{IH} 5)^{13}\end{array}$ & $\begin{array}{c}\text { X-ray bAQP1 }^{(1)} \\
(1 \mathrm{~J} 4 \mathrm{~N})^{14}\end{array}$ & $\begin{array}{c}\text { Refined EM }^{(5)} \\
(1 \mathrm{H} 6)^{37}\end{array}$ & $\begin{array}{c}\text { X-ray } \\
(4 \mathrm{CSK})^{38}\end{array}$ \\
\hline H1 & A13-F35 & K8-Y37 & W11-G34 & F5-Y37 & L9-Y37 & F5-L33 \\
H2 & N49-H69 & D48-V67 & D48-T62 & D50-G74 & N49-I70 & D48-G68 \\
HB & P77-G82 & N76-L85 & N76-C87 & N78-S88 & N76-S86 & N76-S86 \\
H3 & R93-I115 & A94-T116 & L95-T120 & S92-T118 & S90-G114 & F92-T116 \\
H4 & S135-T156 & G136-T156 & L139-R159 & G138-T159 & N134-T157 & G138-T156 \\
H5 & A168-Y186 & G166-I184 & L170-I184 & S169-G190 & S167-I184 & S167-A183 \\
HE & P193-V201 & N192-V201 & P193-I202 & N194-T205 & N192-H204 & N192-G198 \\
H6 & S207-I226 & S207-F229 & W210-D228 & W212-F231 & W210-L231 & W210-F229 \\
\hline
\end{tabular}

(1) Bovine AQP1 numbering. Human AQP1 numbering is shifted down by 2 residues starting from A45.

(2) N-terminus was not detected up to residue A13 in NMR experiments.

(3) No residues detected in the 83-92 stretch.

(4) C-terminus was not detected after residue I226 in NMR experiments.

(5) Refined structural model of hAQP1 based on the EM structure 1FQY and the X-ray structure of its bacterial homolog GlpF. 
Table S2. Target angles and force constants for hAQP1 restrained simulations. Units for positional restraints, $\mathrm{k}_{\varphi}$ and $\mathrm{k}_{\psi}$, are $\mathrm{kJ} / \mathrm{mol} / \mathrm{rad}^{2}$.

\begin{tabular}{|c|c|c|c|c|c|c|c|c|c|c|c|c|}
\hline Res. & $\begin{array}{c}\text { Target } \varphi \\
\text { (deg) }\end{array}$ & $\begin{array}{l}\text { Stdev. } \\
\text { (deg) }\end{array}$ & $\begin{array}{c}\text { Target } \psi \\
\text { (deg) }\end{array}$ & $\begin{array}{l}\text { Stdev. } \\
\text { (deg) }\end{array}$ & $\begin{array}{c}\mathbf{k}_{\varphi} \\
\text { DR } 1\end{array}$ & $\begin{array}{c}\mathbf{k}_{\psi} \\
\text { DR } 1\end{array}$ & $\begin{array}{c}\mathbf{k}_{\varphi} \\
\mathbf{D R} 2\end{array}$ & $\begin{array}{c}\mathbf{k}_{\psi} \\
\text { DR } 2\end{array}$ & $\begin{array}{c}\mathbf{k}_{\varphi} \\
\text { DR } 3\end{array}$ & $\begin{array}{c}\mathbf{k}_{\psi} \\
\text { DR } 3\end{array}$ & $\begin{array}{c}\mathbf{k}_{\varphi} \\
\text { DR } 4\end{array}$ & $\begin{array}{c}\mathbf{k}_{\psi} \\
\text { DR } 4\end{array}$ \\
\hline F35 & -79.90 & 20.60 & -16.48 & 20.05 & 1.21 & 1.25 & 2.43 & 2.49 & 4.85 & 4.99 & 9.71 & 9.97 \\
\hline K36 & -98.06 & 8.20 & 2.03 & 11.69 & 3.05 & 2.14 & 6.10 & 4.28 & 12.20 & 8.56 & 24.41 & 17.11 \\
\hline Y37 & -105.82 & 34.14 & 147.22 & 27.08 & 0.73 & 0.92 & 1.46 & 1.85 & 2.93 & 3.69 & 5.86 & 7.39 \\
\hline P38 & -81.73 & 31.35 & 151.29 & 13.66 & 0.80 & 1.83 & 1.59 & 3.66 & 3.19 & 7.32 & 6.38 & 14.64 \\
\hline T44 & -103.38 & 15.10 & -10.31 & 20.80 & 1.66 & 1.20 & 3.31 & 2.40 & 6.62 & 4.81 & 13.25 & 9.62 \\
\hline A45 & -69.20 & 10.94 & 144.47 & 22.38 & 2.29 & 1.12 & 4.57 & 2.23 & 9.14 & 4.47 & 18.28 & 8.93 \\
\hline V46 & -121.66 & 19.84 & 135.77 & 19.96 & 1.26 & 1.25 & 2.52 & 2.51 & 5.04 & 5.01 & 10.08 & 10.02 \\
\hline Q47 & -97.66 & 8.88 & 117.90 & 11.58 & 2.81 & 2.16 & 5.63 & 4.32 & 11.26 & 8.63 & 22.52 & 17.27 \\
\hline D48 & -93.22 & 20.90 & 131.68 & 31.20 & 1.20 & 0.80 & 2.39 & 1.60 & 4.78 & 3.21 & 9.57 & 6.41 \\
\hline N49 & -55.44 & 5.45 & -36.88 & 7.32 & 4.59 & 3.42 & 9.18 & 6.84 & 18.36 & 13.67 & 36.71 & 27.34 \\
\hline V50 & -61.27 & 3.85 & -36.50 & 11.56 & 6.50 & 2.16 & 12.99 & 4.33 & 25.99 & 8.65 & 51.98 & 17.30 \\
\hline G114 & -61.72 & 6.04 & -41.10 & 4.50 & 4.14 & 5.56 & 8.28 & 11.12 & 16.56 & 22.23 & 33.12 & 44.46 \\
\hline I115 & -65.19 & 11.73 & -31.56 & 9.08 & 2.13 & 2.75 & 4.26 & 5.51 & 8.53 & 11.01 & 17.05 & 22.02 \\
\hline T116 & -87.53 & 24.34 & -15.14 & 20.49 & 1.03 & 1.22 & 2.05 & 2.44 & 4.11 & 4.88 & 8.22 & 9.76 \\
\hline S117 & -75.44 & 23.17 & -35.54 & 9.47 & 1.08 & 2.64 & 2.16 & 5.28 & 4.32 & 10.56 & 8.63 & 21.11 \\
\hline S118 & -75.92 & 16.18 & -15.87 & 19.48 & 1.55 & 1.28 & 3.09 & 2.57 & 6.18 & 5.13 & 12.36 & 10.27 \\
\hline $\mathrm{T} 120$ & -90.97 & 27.43 & 144.61 & 19.85 & 0.91 & 1.26 & 1.82 & 2.52 & 3.65 & 5.04 & 7.29 & 10.08 \\
\hline N122 & -84.65 & 13.41 & -12.75 & 22.51 & 1.86 & 1.11 & 3.73 & 2.22 & 7.45 & 4.44 & 14.91 & 8.89 \\
\hline L124 & -83.10 & 10.59 & -2.93 & 10.70 & 2.36 & 2.34 & 4.72 & 4.67 & 9.44 & 9.34 & 18.88 & 18.69 \\
\hline G125 & 83.48 & 19.08 & 18.44 & 17.55 & 1.31 & 1.42 & 2.62 & 2.85 & 5.24 & 5.70 & 10.48 & 11.40 \\
\hline R126 & -78.08 & 34.42 & 125.30 & 8.13 & 0.73 & 3.08 & 1.45 & 6.15 & 2.91 & 12.30 & 5.81 & 24.61 \\
\hline N127 & 66.23 & 14.24 & 18.13 & 33.10 & 1.76 & 0.76 & 3.51 & 1.51 & 7.02 & 3.02 & 14.05 & 6.04 \\
\hline L129 & -77.21 & 14.03 & 135.50 & 12.88 & 1.78 & 1.94 & 3.56 & 3.88 & 7.13 & 7.76 & 14.25 & 15.53 \\
\hline A 130 & -87.63 & 19.24 & 132.08 & 26.47 & 1.30 & 0.94 & 2.60 & 1.89 & 5.20 & 3.78 & 10.40 & 7.56 \\
\hline D131 & -56.62 & 6.95 & 133.69 & 5.72 & 3.60 & 4.37 & 7.20 & 8.75 & 14.39 & 17.49 & 28.79 & 34.99 \\
\hline V133 & -99.88 & 13.80 & 120.48 & 11.61 & 1.81 & 2.15 & 3.62 & 4.31 & 7.25 & 8.62 & 14.50 & 17.23 \\
\hline N134 & -83.83 & 11.28 & 177.49 & 10.31 & 2.22 & 2.43 & 4.43 & 4.85 & 8.87 & 9.70 & 17.73 & 19.41 \\
\hline S135 & -62.28 & 4.91 & -27.40 & 18.62 & 5.09 & 1.34 & 10.18 & 2.68 & 20.36 & 5.37 & 40.72 & 10.74 \\
\hline G136 & -62.93 & 3.88 & -41.86 & 5.06 & 6.44 & 4.94 & 12.87 & 9.88 & 25.75 & 19.76 & 51.49 & 39.52 \\
\hline Q137 & -60.91 & 7.04 & -37.10 & 13.11 & 3.55 & 1.91 & 7.10 & 3.81 & 14.20 & 7.63 & 28.39 & 15.26 \\
\hline G138 & -64.74 & 8.70 & -28.94 & 26.35 & 2.87 & 0.95 & 5.75 & 1.90 & 11.49 & 3.79 & 22.99 & 7.59 \\
\hline
\end{tabular}




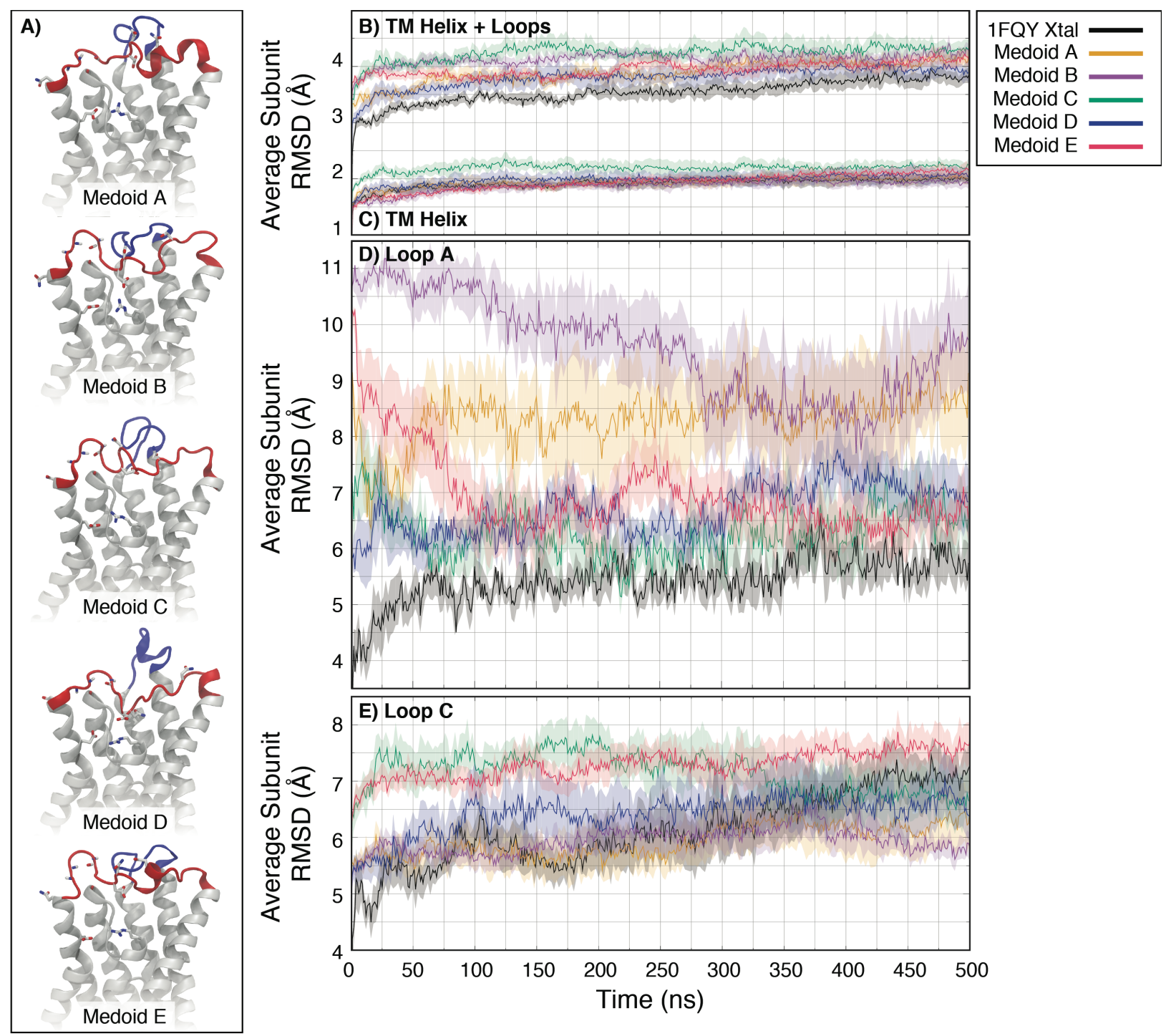

Figure S4. Molecular rendering of the five cluster medoids and structural deviation over time. (A) An overlay of all structures in side views (omitting residues 196-215 for visual clarity) are shown with key side chains of residues N127, D128, E142, and R195 in addition to backbone nitrogen atoms of D131, V133, and N134. Loop A is shown in blue and loop C is shown in red. (C-F) Average root mean square deviation (RMSD) from the EM structure throughout unrestrained simulations of each hAQP1 system. Mean and error bars (standard error of mean) were calculated using the $\mathrm{C} \alpha$ atoms of all monomers of both simulation repeats. (C-D) structural deviation of the full protein structure and transmembrane helices only (defined as residues 8-36, 49-66, 77-84, 95-115, 137-155, 167-183, 193-200, and 208-228). (E) structural deviation of loop A (defined as residues 37-48) and (F) structural deviation of loop C (defined as residues 116 to 136). 

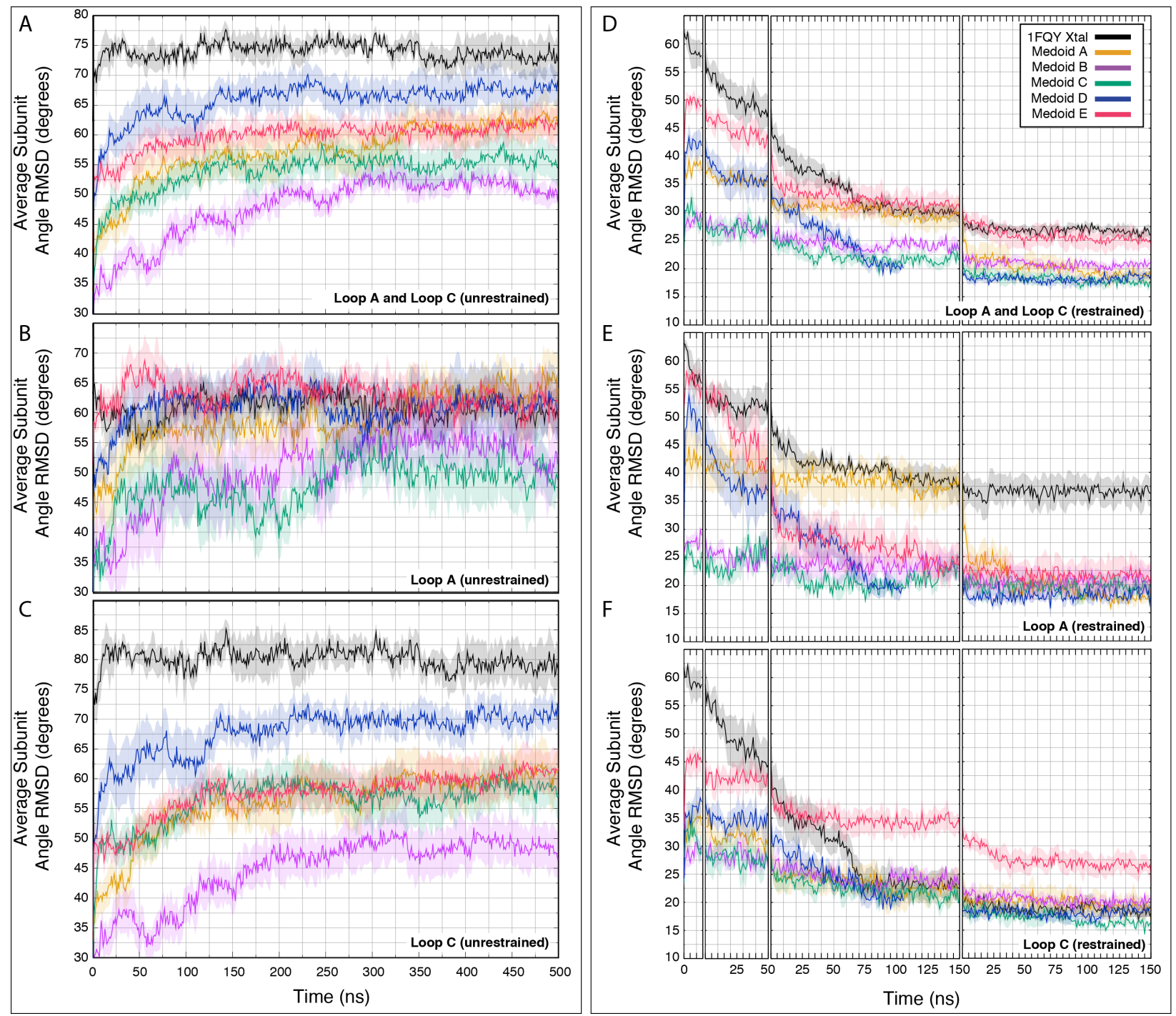

Figure S5. Average angular root mean square deviation (RMSD) from the TALOS + target phi/psi angles throughout unrestrained and restrained simulations of each hAQP1 system. Mean and error bars (standard error of mean) were calculated using all monomers of both simulation repeats. Time series for unrestrained simulations are shown for (A) the angular deviation of loop A and loop C combined, (B) the angular deviation of only loop A, and (C) the angular deviation of loop C. These panels are reproduced for the first $365 \mathrm{~ns}$ of restrained simulations (D-F). Note that restrained simulations have a different y-axis range compared to unrestrained simulations. 

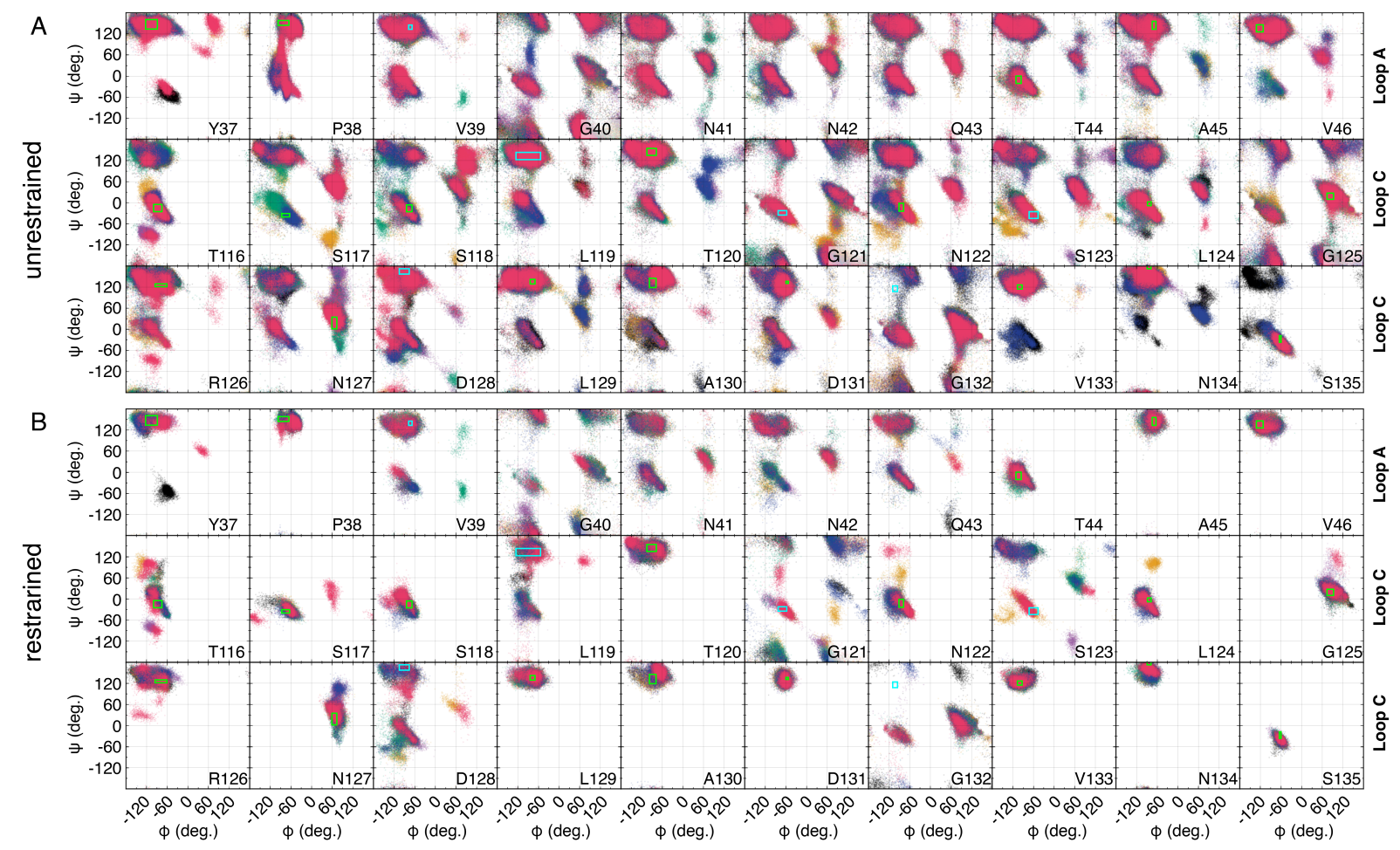

Figure S6. Ramachandran plots for loop A and loop C residues from molecular dynamics simulations. Target $\varphi / \psi$ angle pairs are shown as coloured boxes with width and height determined by the standard deviation of TALOS+ predictions. Green boxes indicate residues with TALOS+ predictions classified as "Good" and where torsional restraints were applied, and cyan boxes indicate residues with TALOS+ predictions classified as "Warning" and torsional restraints were not applied. $\varphi / \psi$ pairs from the EM structure, and medoid A, B, C, D, E models are represented as black, yellow, purple, green, blue, and red points respectively. An equivalent number of frames were analyzed for each simulation system, with angles plotted sequentially in the aforementioned order, resulting in red points being the most visually apparent. 

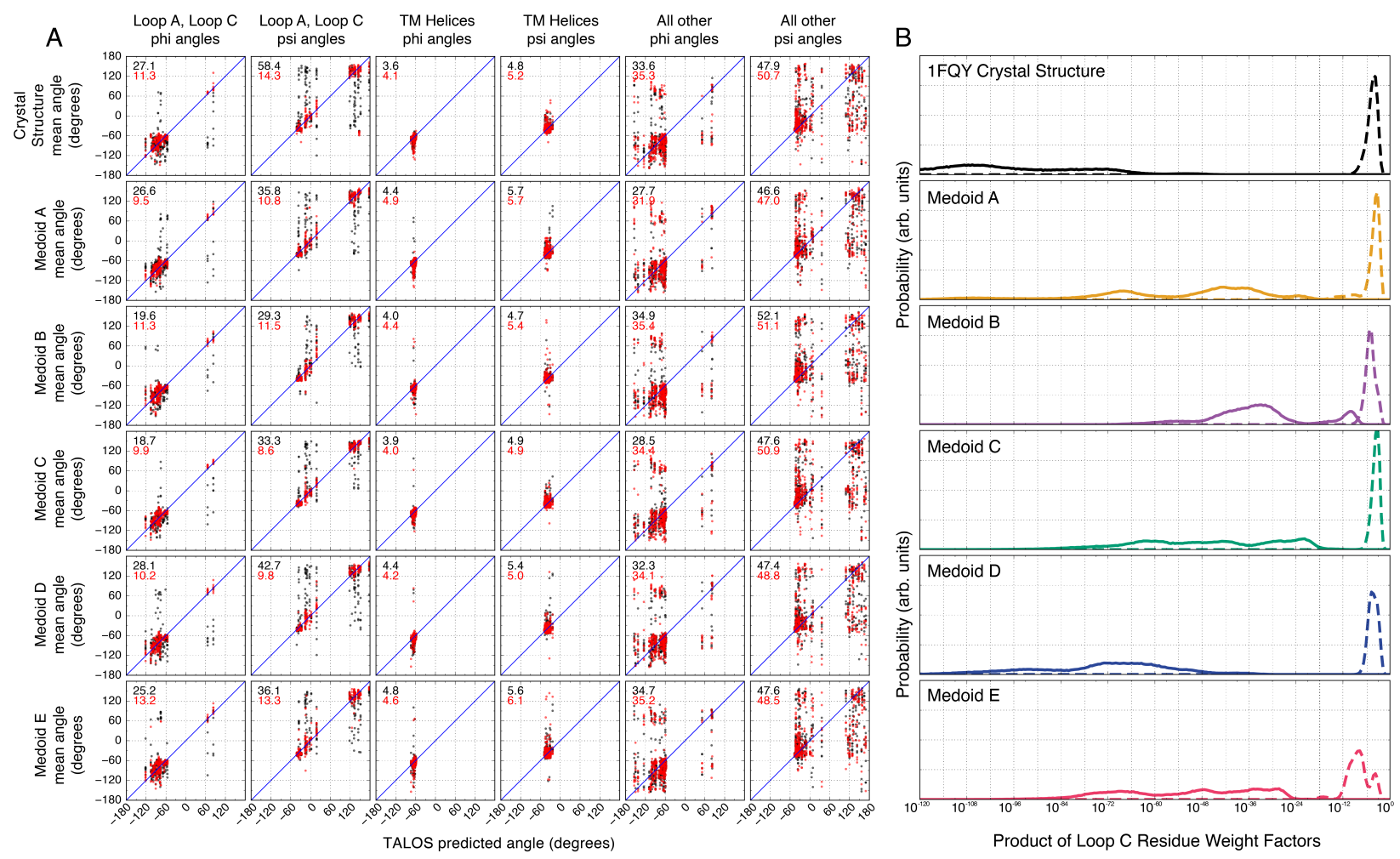

Figure S7. (A) Average deviations in $\varphi / \psi$ angles between unrestrained (black) and restrained simulations (red) to TALOS+ predictions for each monomer of each hAQP1 system. Average angular deviation of all points to TALOS+ predictions is shown in the top left for both unrestrained and restrained data. (B) Distributions of the product of exponential weight factors for loop C phi/psi angles in unrestrained (solid lines) and restrained datasets (dashed lines). For each monomer of both repeats of a given system, all phi/psi angles were assigned an exponential weighting factor based on the deviation to TALOS + target and the dihedral angle force constant for that angle. The distribution of the product of these factors for all frames of each model is shown. 


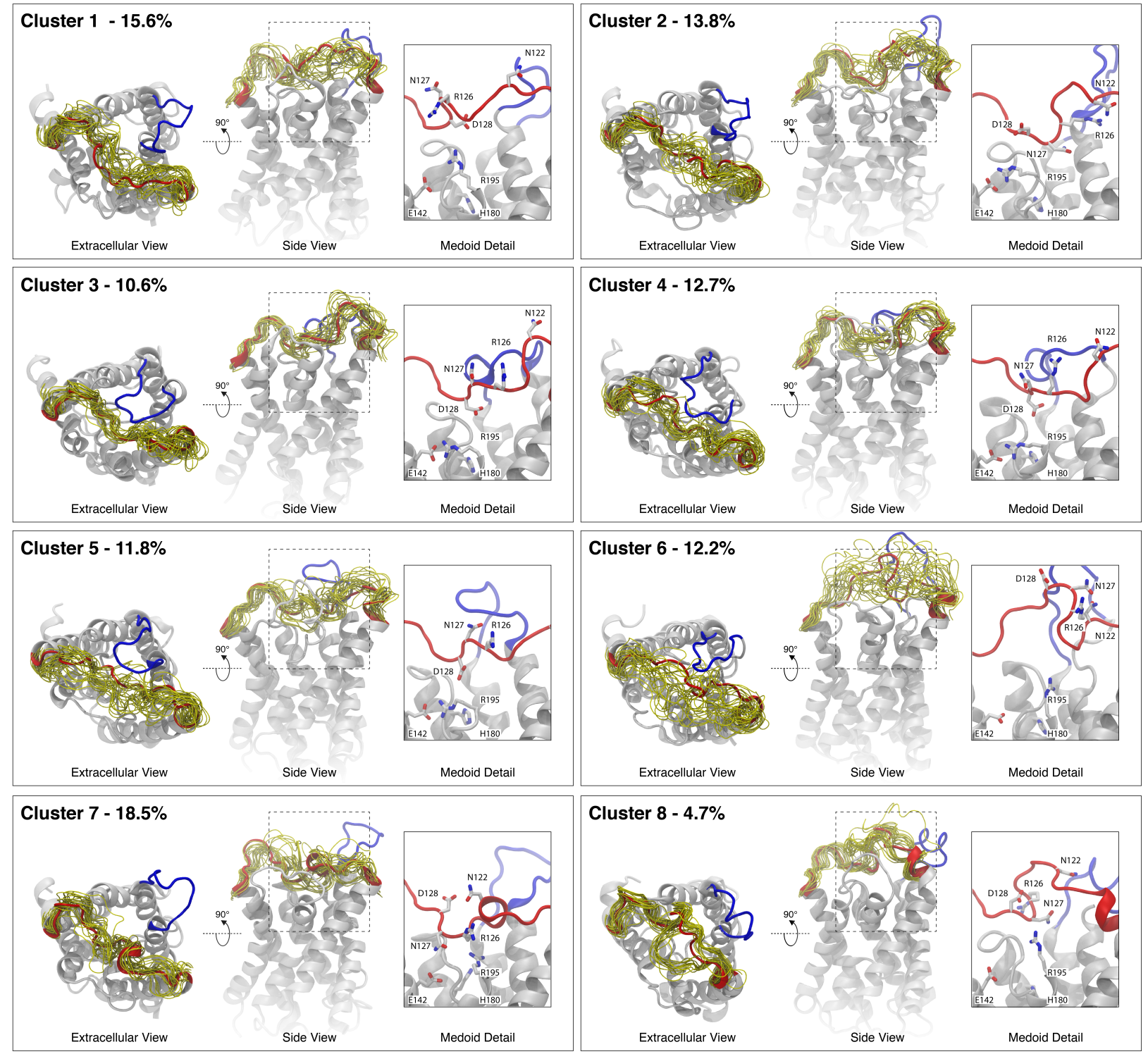

Figure S8. Molecular renderings of multiple frames from RMSD-based clustering of loop C (Figure 8) in the restrained dataset. Single snapshots of the transmembrane regions are shown with overlays of loop A (blue) and loop C (red) from the medoid structures of each cluster. Yellow traces represent the backbone of twenty loop $\mathrm{C}$ conformations found within each cluster. Medoid detail describes the intermolecular interactions of loop $\mathrm{C}$ and $\mathrm{ar} / \mathrm{R}$ filter side chains described in the H/D exchange experiments section (R126, N127, D128, E142, H180, R195). 


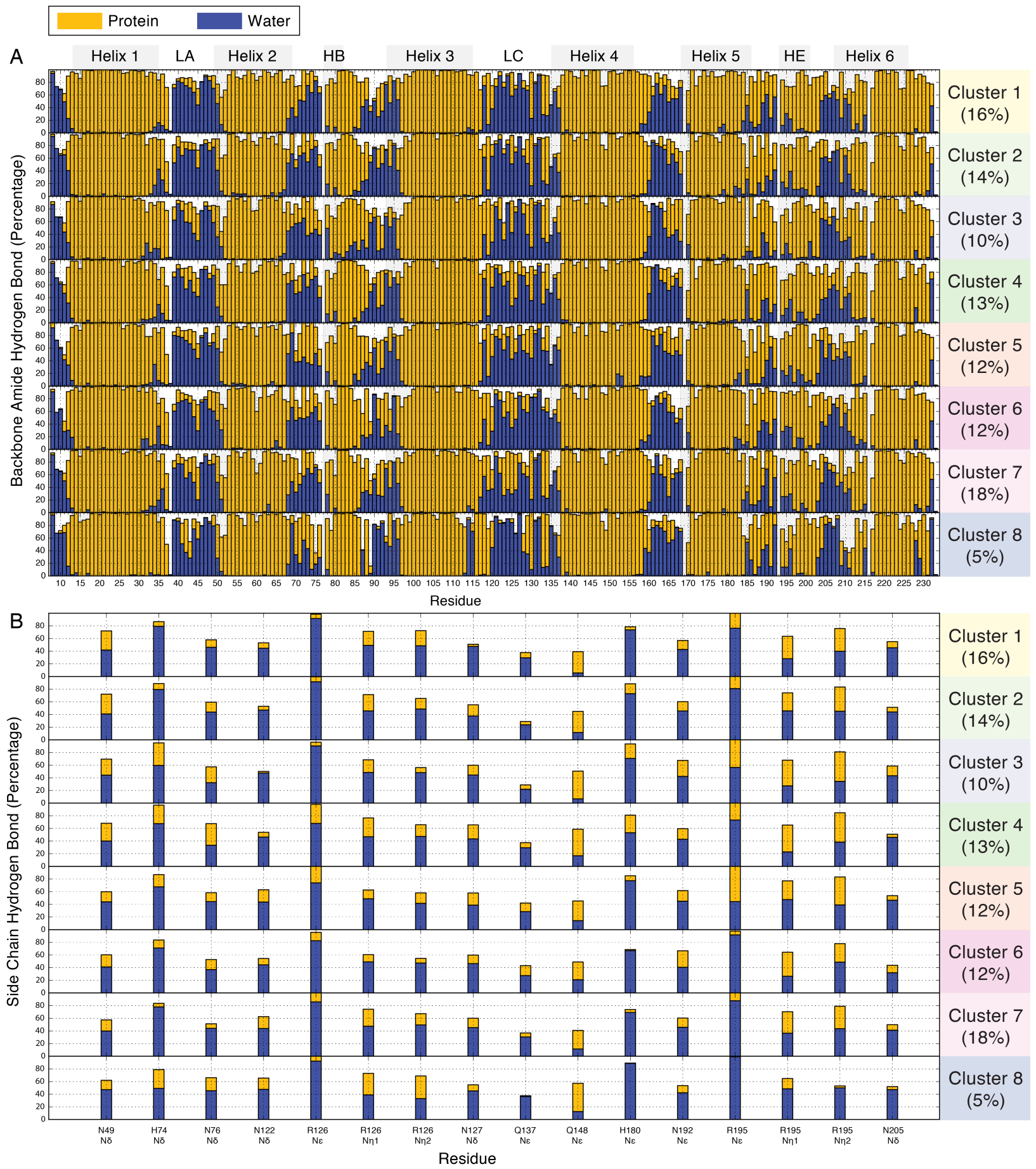

Figure S9. Percentage of restrained dataset where nitrogen atoms of each residue were hydrogen bonded to protein or water oxygen atoms. Percentage is computed for each cluster obtained from K-Medoids clustering of loop C conformations. (A) Water and protein hydrogen bond percentage for backbone nitrogen atoms. (B) Water and protein hydrogen bond percentage for key nitrogen side chains. 

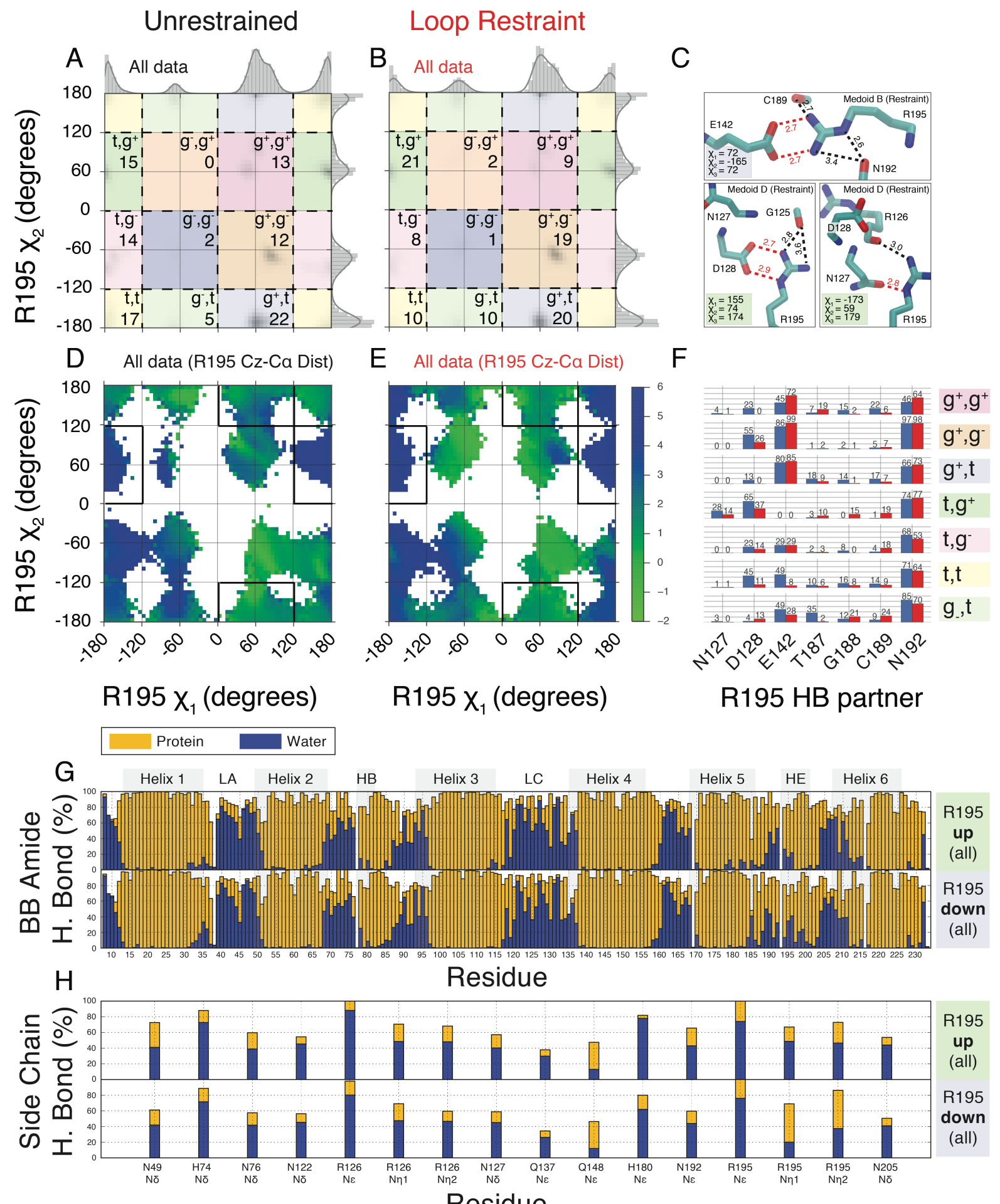

Figure S10. Conformational states of R195 in unrestrained and restrained simulations. (A-B) 2D histogram of R195 $\chi_{1}$ and $\chi_{2}$ with populations of conformational basins. (C) Representative 
snapshots of hydrogen bonding partners of R195 in up-facing and side-facing conformations. (DE) $2 \mathrm{D}$ histogram of R195 $\chi_{1}$ and $\chi_{2}$ with histogram value representing the average $z$-axis distance of $\mathrm{Cz}$ to $\mathrm{C} \alpha$ at that point. (F) Percentage of frames where R195 (either backbone or side chain) forms a hydrogen bond with a neighbouring residue, calculated separately for each conformational basin in $\chi_{1}$ and $\chi_{2}$ space, for unrestrained (black) and restrained data (red). (G-H) Percentage of restrained dataset where backbone or side chain nitrogen atoms were hydrogen bonded to protein or water oxygen atoms. Percentage is computed for the subset of restrained data where R195 adopted $\left(\mathrm{t}, \mathrm{g}^{+}\right)$and $\left(\mathrm{g}^{+}, \mathrm{t}\right) \chi_{1}, \chi_{2}$ basins, corresponding to an up-facing and sidefacing state, respectively.

\section{References}

(1) Emami, S.; Fan, Y.; Munro, R.; Ladizhansky, V.; Brown, L. S. Yeast-Expressed Human Membrane Protein Aquaporin-1 Yields Excellent Resolution of Solid-State MAS NMR Spectra. J Biomol NMR 2013, 55, 147-155.

(2) Fan, Y.; Emami, S.; Munro, R.; Ladizhansky, V.; Brown, L. S. Isotope Labeling of Eukaryotic Membrane Proteins in Yeast for Solid-State NMR. In Isotope Labeling of Biomolecules - Labeling Methods; Kelman, Z., Ed.; Methods in Enzymology; Academic Press, 2015; Vol. Volume 565, pp 193-212.

(3) Nyblom, M.; Öberg, F.; Lindkvist-Petersson, K.; Hallgren, K.; Findlay, H.; Wikström, J.; Karlsson, A.; Hansson, Ö.; Booth, P. J.; Bill, R. M.; et al. Exceptional Overproduction of a Functional Human Membrane Protein. Protein Expression and Purification 2007, 56, 110-120.

(4) Baldus, M.; Petkova, A. T.; Herzfeld, J.; Griffin, R. G. Cross Polarization in the Tilted Frame: Assignment and Spectral Simplification in Heteronuclear Spin Systems. Mol. Phys. 1998, 95, 1197-1207.

(5) Fung, B. M.; Khitrin, A. K.; Ermolaev, K. An Improved Broadband Decoupling Sequence for Liquid Crystals and Solids. Journal of Magnetic Resonance 2000, 142, 97 101.

(6) Morcombe, C. R.; Zilm, K. W. Chemical Shift Referencing in MAS Solid State NMR. Journal of Magnetic Resonance 2003, 162, 479-486.

(7) Delaglio, F.; Grzesiek, S.; Vuister, G. W.; Zhu, G.; Pfeifer, J.; Bax, A. NMRPipe: a Multidimensional Spectral Processing System Based on UNIX Pipes. J Biomol NMR 1995, 6, 277-293.

(8) Keller, R. The Computer Aided Resonance Assignment Tutorial, 1st ed.; CANTINA Verlag : Goldau, 2004; pp 1-73.

(9) Takegoshi, K.; Nakamura, S.; Terao, T. 13C-1H Dipolar-Assisted Rotational Resonance in Magic-Angle Spinning NMR. Chem. Phys. Lett. 2001, 344, 631-637.

(10) Murata, K.; Mitsuoka, K.; Hirai, T.; Walz, T.; Agre, P.; Heymann, J. B.; Engel, A.; Fujiyoshi, Y. Structural Determinants of Water Permeation Through Aquaporin-1. Nature 2000, 407, 599-605.

(11) Fiser, A.; Šali, A. Modeller: Generation and Refinement of Homology-Based Protein Structure Models. Methods in enzymology 2003, 374, 461-491. 
(12) Jamroz, M.; Kolinski, A. ClusCo: Clustering and Comparison of Protein Models. BMC Bioinformatics 2013, 14, 62.

(13) Ren, G.; Reddy, V. S.; Cheng, A. Visualization of a Water-Selective Pore by Electron Crystallography in Vitreous Ice. Proc. Natl. Acad. Sci. U. S. A. 2001, 98, 1398-1403.

(14) Sui, H.; Han, B.-G.; Lee, J. K.; Walian, P.; Jap, B. K. Structural Basis of Water-Specific Transport Through the AQP1 Water Channel. Nature 2001, 414, 872-878.

(15) Humphrey, W.; Dalke, A.; Schulten, K. VMD: Visual Molecular Dynamics. J. Mol. Graphics 1996, 14, 33-38.

(16) Schmidt, T. H.; Kandt, C. LAMBADA and InflateGRO2: Efficient Membrane Alignment and Insertion of Membrane Proteins for Molecular Dynamics Simulations. $J$. Chem. Inf. Model. 2012, 52, 2657-2669.

(17) Wolf, M. G.; Hoefling, M.; Aponte-Santamaría, C.; Grubmüller, H.; Groenhof, G. G_Membed: Efficient Insertion of a Membrane Protein Into an Equilibrated Lipid Bilayer with Minimal Perturbation. J. Comp. Chem. 2010, 31, 2169-2174.

Pronk, S.; Pall, S.; Schulz, R.; Larsson, P.; Bjelkmar, P.; Apostolov, R.; Shirts, M. R.;

Smith, J. C.; Kasson, P. M.; van der Spoel, D.; et al. GROMACS 4.5: a High-

Throughput and Highly Parallel Open Source Molecular Simulation Toolkit. Bioinformatics 2013, 29, 845-854.

(19) Best, R. B.; Zhu, X.; Shim, J.; Lopes, P. E.; Mittal, J.; Feig, M.; MacKerell, A. D., Jr. Optimization of the Additive CHARMM All-Atom Protein Force Field Targeting Improved Sampling of the Backbone $\Phi, \Psi$ and Side-Chain X1 and X2 Dihedral Angles. J. Chem. Theory Comput. 2012, 8, 3257-3273.

(20) Klauda, J. B.; Venable, R. M.; Freites, J. A. Update of the CHARMM All-Atom Additive Force Field for Lipids: Validation on Six Lipid Types. J. Phys. Chem. B 2010, 114, 7830-7843.

(21) Jorgensen, W.; Chandrasekhar, J.; Madura, J. D.; Impey, R. W.; Klein, M. Comparison of Simple Potential Functions for Simulating Liquid Water. J. Chem. Phys. 1983, 79, 926-935.

(22) Darden, T.; York, D.; Pedersen, L. Particle Mesh Ewald: an Nlog(N) Method for Ewald Sums in Large Systems. J. Chem. Phys. 1993, 98, 10089-10092.

(23) Essmann, U.; Perera, L.; Berkowitz, M. L.; Darden, T.; Lee, H.; Pederson, L. G. A Smooth Particle Mesh Ewald Method. J. Chem. Phys. 1995, 103, 8577-8593.

(24) Nosé, S.; Klein, M. L. Constant Pressure Molecular Dynamics for Molecular Systems. Mol. Phys. 1983, 50, 1055-1076.

(25) Nosé, S. A Unified Formulation of the Constant Temperature Molecular-Dynamics Methods. J. Chem. Phys. 1984, 81, 511-519.

(26) Hoover, W. G. Canonical Dynamics: Equilibrium Phase-Space Distributions. Phys. Rev. A 1985, 31, 1695.

(27) Miyamoto, S.; Kollman, P. A. SETTLE: an Analytical Version of the SHAKE and RATTLE Algorithm. J. Comp. Chem. 1992, 13, 952-962.

(28) Hess, B. P-LINCS: a Parallel Linear Constraint Solver for Molecular Simulation. $J$. Chem. Theory Comput. 2008, 4, 116-122.

(29) Zhu, F.; Tajkhorshid, E.; Schulten, K. Collective Diffusion Model for Water Permeation Through Microscopic Channels. Phys. Rev. Lett. 2004, 93, 224501.

(30) Michaud-Agrawal, N.; Denning, E. J.; Woolf, T. B.; Beckstein, O. MDAnalysis: a Toolkit for the Analysis of Molecular Dynamics Simulations. J. Comp. Chem. 2011, 32, 
2319-2327.

(31) McGibbon, R. T.; Beauchamp, K. A.; Harrigan, M. P.; Klein, C.; Swails, J. M.; Hernández, C. X.; Schwantes, C. R.; Wang, L.-P.; Lane, T. J.; Pande, V. S. MDTraj: a Modern Open Library for the Analysis of Molecular Dynamics Trajectories. Biophys. J. 2015, 109, 1528-1532.

(32) Kabsch, W.; Sander, C. Dictionary of Protein Secondary Structure: Pattern Recognition of Hydrogen-Bonded and Geometrical Features. Biopolymers 1983, 22, 2577-2637.

(33) Beauchamp, K. A.; Bowman, G. R.; Lane, T. J.; Maibaum, L.; Haque, I. S.; Pande, V. S. MSMBuilder2: Modeling Conformational Dynamics on the Picosecond to Millisecond Scale. J. Chem. Theory Comput. 2011, 7, 3412-3419.

(34) Hub, J. S.; Aponte-Santamaría, C.; Grubmüller, H.; de Groot, B. L. Voltage-Regulated Water Flux Through Aquaporin Channels in Silico. Biophys. J. 2010, 99, L97-L99.

(35) Grossfield, A.; Feller, S. E.; Pitman, M. C. Convergence of Molecular Dynamics Simulations of Membrane Proteins. Proteins 2007, 67, 31-40.

(36) Boonstra, S.; Onck, P. R.; Giessen, E. V. D. CHARMM TIP3P Water Model Suppresses Peptide Folding by Solvating the Unfolded State. J. Phys. Chem. B 2016, 120, 36923698.

(37) de Groot, B. L.; Engel, A.; Grubmüller, H. A Refined Structure of Human Aquaporin-1. FEBS Lett. 2001, 504, 206-211.

(38) Ruiz Carrillo, D.; To Yiu Ying, J.; Darwis, D.; Soon, C. H.; Cornvik, T.; Torres, J.; Lescar, J. Crystallization and Preliminary Crystallographic Analysis of Human Aquaporin 1 at a Resolution of $3.28 \AA$ A. Acta Crystallogr F Struct Biol Commun 2014, 70, 1657-1663. 